\title{
The utility of PAX5 immunohistochemistry in the diagnosis of undifferentiated malignant neoplasms
}

\author{
Kristin C Jensen ${ }^{1,2}$, John PT Higgins ${ }^{1}$, Kelli Montgomery ${ }^{1}$, Gulsah Kaygusuz ${ }^{3}$, \\ Matt van de Rijn ${ }^{1}$ and Yasodha Natkunam ${ }^{1}$ \\ ${ }^{1}$ Department of Pathology, Stanford University School of Medicine, Stanford, CA, USA; ${ }^{2}$ Department of \\ Pathology, Veterans Affairs Palo Alto Health Care System, Palo Alto, CA, USA and ${ }^{3}$ Department of Pathology, \\ Ankara University, School of Medicine, Ankara, Turkey
}

\begin{abstract}
PAX5 is a B-cell transcription factor whose expression at the protein level is reliably detected by immunohistochemistry in routine biopsies. The purpose of this study was to investigate whether PAX5 immunohistochemistry has diagnostic benefit as a B-cell marker in the work-up of undifferentiated malignant neoplasms. Twenty-five cases previously diagnosed as undifferentiated malignant neoplasms were selected. In addition, 59 hematolymphoid and 884 non-hematolymphoid malignancies were studied such that the specificity of PAX5 immunohistochemistry could be addressed. Two of the $25(8 \%)$ undifferentiated neoplasms showed diffuse staining for PAX5, which indicated a B-cell derivation for these neoplasms that was not appreciated at the time of initial diagnosis. PAX5 staining was detected in the vast majority of hematolymphoid tumors of Bcell derivation but only in 5 of 884 (less than 1\%) non-hematolymphoid tumors. Our results further show that PAX5 may be the only detectable marker of B lineage in lymphomas that lack or show equivocal CD45RB and CD20 expression. We conclude that the addition of PAX5 to a panel of immunohistologic markers used in the interrogation of undifferentiated neoplasms is of diagnostic benefit. Its expression can also facilitate the diagnosis of classical and nodular lymphocyte-predominant Hodgkin lymphoma with atypical morphologic and immunohistologic features. Lastly, we have shown that the lack of its expression at the protein level in many epithelial and mesenchymal neoplasms renders PAX5 expression an extremely specific marker of the B lineage. Modern Pathology (2007) 20, 871-877; doi:10.1038/modpathol.3800831; published online 25 May 2007
\end{abstract}

Keywords: PAX5; B cell; non-Hodgkin lymphoma; Hodgkin lymphoma; undifferentiated neoplasm

$P A X 5$ (B-cell-specific activator protein, $B S A P$ ) is a member of the paired box domain gene family that encodes nuclear transcription factors important in development, differentiation, cell migration and proliferation. ${ }^{1,2}$ PAX5 protein is expressed as a nuclear marker in B-lineage cells that span the differentiation spectrum from precursor B cells to early plasma cells. ${ }^{3,4}$ The expression of PAX5 protein is also a useful lineage-specific marker in hematopoietic neoplasms arising from B cells. ${ }^{5-8}$ Staining for PAX5 has additional utility in the

Correspondence: Dr KC Jensen, MD, Department of Pathology, Stanford University School of Medicine, Room L235, 300 Pasteur Drive, Stanford, CA 94305, USA.

E-mail: kjensen1@stanford.edu

The findings in this manuscript were originally presented as two separate posters in 2005 at the 94th Annual Meeting of the United States and Canadian Academy of Pathology, San Antonio, TX, USA.

Received 7 March 2007; accepted 18 April 2007; published online 25 May 2007 diagnosis of B-cell malignancies that lack expression of commonly used pan-B-cell markers such as CD20 and CD79a. ${ }^{9}$ In addition to its expression in B cells, PAX5 protein and/or mRNA has been reported to be expressed in normal and neoplastic cell types in the central nervous system, testis and bladder.,10-15

B-cell malignancies may express PAX5 in the absence of expression of other pan-B-cell markers, suggesting that the inclusion of PAX5 in a panel of antibodies to assess undifferentiated malignant neoplasms may have diagnostic benefit. To address this possibility, we studied examples previously diagnosed as undifferentiated malignant neoplasms for PAX5 expression by immunohistologic methods. Hodgkin and non-Hodgkin lymphomas (NHLs) that lacked or showed equivocal staining for CD20 were also included. Using tissue microarrays (TMA) and conventional paraffin sections of a large number of epithelial and mesenchymal tumors, we further investigated the tissue distribution pattern of PAX5 expression to address the specificity of PAX5 
staining in various cell and tissue types. Thus, the two aims of the current study were to explore the benefit of adding PAX5 to the immunohistologic panel used for the interrogation of undifferentiated malignant neoplasms and the specificity of PAX5 immunostaining in the separation of B-cell-derived neoplasms from their morphologic mimics.

\section{Materials and methods}

\section{Selection of Cases and TMA}

A total of 968 cases identified from the Department of Pathology archives of Stanford University Medical Center comprise this study. Twenty-five cases were selected which had been previously diagnosed as 'undifferentiated' malignant neoplasms based on the lack of lineage-specific immunohistochemical staining; sufficient material for additional immunohistochemical stains was available (Table 1). Fiftyseven hematopoietic tumors exhibiting unusual immunophenotypic features were also selected and included four NHLs with weak, partial or lack of CD20 expression, 45 classical Hodgkin lymphomas (CHL) and eight nodular lymphocyte-predominant Hodgkin lymphomas (NLPHLs) (Tables 2 and 3). CHL, NLPHL and NHL with an immunostaining profile typical of the diagnoses ${ }^{16}$ were excluded from the study. Two acute myeloid leukemias with $\mathrm{t}(8 ; 21)$ were also included.

To investigate the specificity of PAX5, a TMA containing 515 epithelial and mesenchymal neoplasms as well as 9 normal control tissues was utilized (Table 4). A second TMA containing 339 bladder tumors (325 of which were transitional cell carcinomas of varying histologic grades) was also stained for PAX5. Conventional sections from 17 cases of Merkel cell carcinoma, 4 cases of neuroblastoma, 2 cases of medulloblastoma and 7 cases of astrocytoma were also studied. TMAs were constructed in the usual fashion, ${ }^{17}$ assembling $0.6 \mathrm{~mm}$ formalin-fixed, paraffin-embedded tissue cores from donor tissue blocks using a tissue arrayer (Beecher Instruments, Silver Spring, MD, USA).

\section{Immunohistochemistry}

All conventional and TMA sections were cut at $5-\mu$ thickness and baked for a minimum of $60 \mathrm{~min}$ at $60^{\circ} \mathrm{C}$ in a standard histology oven to cause the tissue sections to adhere to charged glass slides. Sections were then subjected to heat-induced epitope retrieval in a citrate $6.0 \mathrm{pH}$ buffer using a pressure cooker for $30 \mathrm{~min}$ (maximum temperature of $115^{\circ} \mathrm{C}$ ), followed by a $20 \mathrm{~min}$ cooling period at room temperature. Sections were stained with anti-PAX5 (clone 24, BD Transduction, Franklin Lakes, NJ, USA) using a Dako autostainer (Dako Cytomation, Carpinteria, CA, USA), and were subsequently counter-stained with hematoxylin and cover-slipped using an automated plastic cover-slipping process (Sakura, McGaw Park, IL, USA).

Table 3 Hodgkin lymphomas with unusual immunophenotypic features

\begin{tabular}{lc}
\hline Immunophenotype & $\begin{array}{l}\text { Number } \\
\text { of cases }\end{array}$ \\
\hline $\begin{array}{l}\text { Classic Hodgkin lymphoma (all CD30-positive) } \\
\text { CD15-negative (six cases CD20-positive; two }\end{array}$ & 44 \\
cases CD45-positive) & 1 \\
CD3-positive & \\
Nodular lymphocyte-predominant Hodgkin lymphoma & 2 \\
CD20-rare or negative & 1 \\
Increased proliferation rate & 1 \\
CD45-negative & 1 \\
Focal nodules of B cells & 1 \\
No ringing by CD57-positive cells & 1 \\
CD30-positive & 1 \\
\hline
\end{tabular}

Table 1 PAX5 immunostaining in undifferentiated malignant neoplasms

\begin{tabular}{|c|c|c|c|c|c|}
\hline Diagnosis & PAX5 & Keratin & $S 100$ & Desmin & $C D 34$ \\
\hline Undifferentiated neoplasm & Positive & Negative (AE1, CAM5.2, EMA) & Negative & Negative & Negative \\
\hline $\begin{array}{l}\text { Undifferentiated neoplasm, consistent } \\
\text { with sarcoma (see Figure 1) }\end{array}$ & Positive & Negative (AE1/CAM5.2 mix) & Negative & Not done & Not done \\
\hline
\end{tabular}

EMA, epithelial membrane antigen.

Table 2 PAX5 staining in non-Hodgkin lymphomas with loss or partial expression of CD20

\begin{tabular}{|c|c|c|c|c|c|c|c|}
\hline Case & $P A X 5$ & $C D 20$ & CD30 & CD15 & $C D 45$ & Mast cell tryptase & Diagnosis \\
\hline 1 & Positive & Negative & Negative & Negative & Positive & Not done & DLBCL \\
\hline 2 & Positive & Subset positive & Not done & Not done & Not done & Not done & DLBCL \\
\hline 3 & Positive & Subset positive & Positive & Not done & Positive & Not done & MLBCL \\
\hline 4 & Positive & Subset positive & Not done & Not done & Not done & Negative & DLBCL \\
\hline
\end{tabular}

CD45, CD45RB (leukocyte common antigen); DLBCL, diffuse large B-cell lymphoma; MLBCL, mediastinal large B-cell lymphoma. 
Two pathologists reviewed the immunostains (KCJ, YN). Nuclear reactivity was considered positive and was scored as focal or diffuse, and weak, moderate or strong. Tissue cores on the TMA sections were not scored if there was no unequivocal tumor, insufficient tissue to interpret or significant grid misalignment on the TMA section.

\section{Results}

Two of the $25(8 \%)$ undifferentiated neoplasms showed moderate-to-strong PAX5 nuclear staining (Figure 1 and Table 1). At the time of initial

Table 4 Summary of cases studies by tissue microarray analysis

\begin{tabular}{lc}
\hline Type of malignancy & $\begin{array}{c}\text { Number of } \\
\text { tissue } \text { cores }^{\mathrm{a}}\end{array}$ \\
\hline Epithelial & $467(463)$ \\
Adenocarcinoma, various sites & $289(285)$ \\
Carcinoma, various sites & 80 \\
Neuroendocrine tumor, various sites & 7 \\
Squamous cell carcinoma, various sites & 61 \\
Transitional cell carcinoma, various sites & 30 \\
& \\
Mesenchymal & $56(52)$ \\
Carcinosarcoma, uterus & 1 \\
Endometrial stromal sarcoma & 3 \\
Germ cell tumor, ovary and testis & 13 \\
Hepatoblastoma & 1 \\
Malignant mesothelioma, lung & 5 \\
Melanoma & $26(22)$ \\
Neuroblastoma & 3 \\
Thymoma & 1 \\
Wilms' tumor & 3 \\
Normal control tissue & \\
Lymph node & $33(9)$ \\
Muscle & $4(2)$ \\
Placenta & $4(2)$ \\
Spleen & $19(2)$ \\
Tonsil & $2(1)$ \\
& $4(2)$
\end{tabular}

${ }^{\mathrm{a}}$ Number of cases listed in parenthesis if different from the number of tissue cores. diagnosis, both cases lacked staining for keratin and S100; however, on review some reactivity with CD20 and CD45 was detected although this staining was not well localized in one case (needle core biopsy), and was weak and patchy in the second, leading to interpretation as nonspecific staining at the time of initial diagnosis.

All four NHLs showed diffuse, moderate-to-strong PAX5 staining (Figure 2 and Table 2). Two of these cases completely lacked staining for CD20, whereas the other two showed weak/partial CD20 staining with poor localization. PAX5 staining indicated that these atypical lymphoid infiltrates were of $\mathrm{B}$ lineage.

Thirty-nine of $45(87 \%)$ CHL and 7 of $8(88 \%)$ NLPHL showed weak-to-moderate PAX5 staining (Figure 3). Since only those with an atypical immunophenotypic profile were included as per our selection criteria (Table 3), the faint PAX5 nuclear expression in CHL compared with that of NLPHL and DLBCL allowed for clear recognition of the Hodgkin cells and aided in the separation of these entities. As expected, the two acute myeloid leukemias exhibiting $t(8 ; 21)$ lacked PAX5 staining.

None of the 515 epithelial or mesenchymal tumors showed PAX5 staining (Table 4). Two of the 339 bladder carcinomas exhibited weak staining for PAX5; one was a well-differentiated transitional cell carcinoma, the other a poorly differentiated transitional cell carcinoma. Three of $17(18 \%)$ cases of Merkel cell carcinoma showed moderate-to-strong PAX5 staining in tumor cells, and no staining was observed in the four neuroblastomas, two medulloblastomas and seven astrocytomas.

\section{Discussion}

Immunohistologic markers that have the ability to identify the line of differentiation (cell of origin) of a neoplastic proliferation are among the most useful in clinical practice. ${ }^{18}$ This tenet has been reinforced

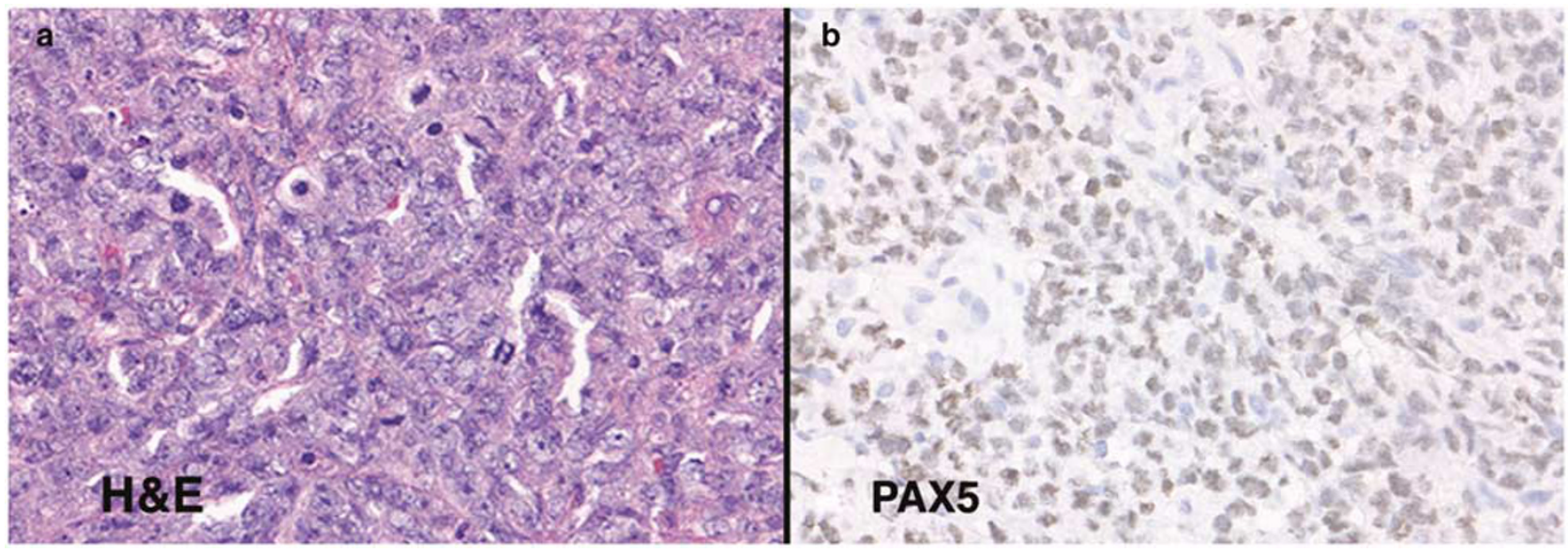

Figure 1 Moderate-to-strong PAX5 reactivity in one of the two undifferentiated neoplasms positive for PAX5 (a, H\&E; b, PAX5). This neoplasm was from a maxillary mass in a 63-year-old male (original magnification $\times 200$ ). 


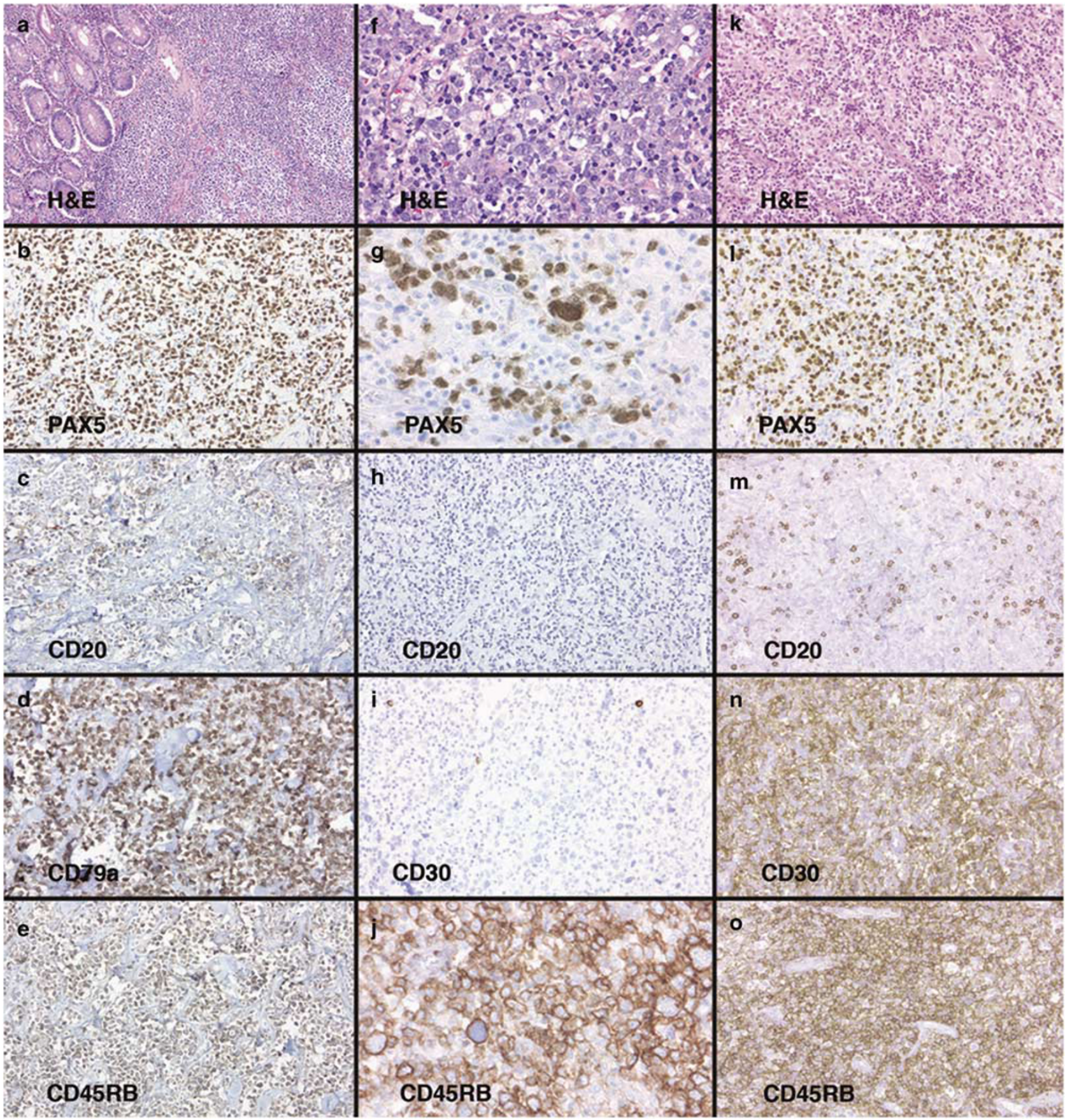

Figure 2 (a-e) and ( $\mathbf{f}-\mathbf{j})$ Representative fields of denoted antibody stains in two of three cases of CD20-negative to -weak diffuse large B-cell lymphoma. ( $\mathbf{k}-\mathbf{o})$ Highlight representative fields of denoted antibody stains in a case of CD20-negative mediastinal large B-cell lymphoma (original magnification $\times 200$ ).

in B-cell neoplasia by gene expression profiling studies which show that B-cell lymphomas tend to maintain the gene expression signature of the stage of differentiation from which they derive. ${ }^{19-21}$ PAX5 is required to maintain B-lymphocyte identity ${ }^{22,23}$ and its expression suppresses the expression of genes that orchestrate differentiation along other lineages. ${ }^{24}$ Coupled with its important role in Blineage identity, the findings in this study confirm that it is also a robust and reliable immunohistologic marker whose tissue-specific expression in B cells can be exploited in routine surgical pathology diagnosis.

The two cases of 'undifferentiated neoplasms' in which we detected PAX5 expression posed many challenges at the time of original diagnosis. The first case represented a small needle core biopsy of a pathologic humeral fracture in a 41-year-old male, 


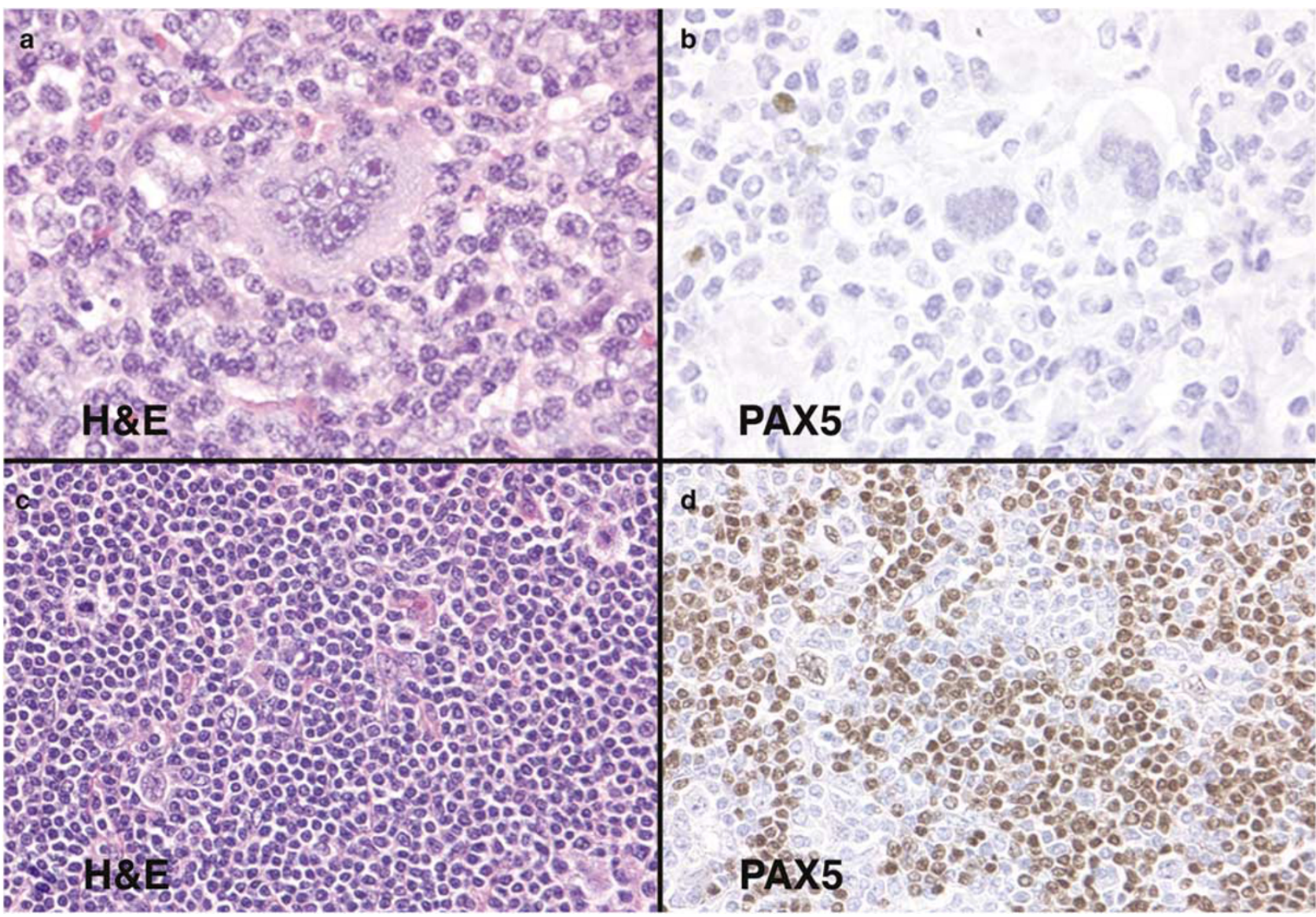

Figure 3 Panels a (H\&E) and b (PAX5) show a case of CHL with weak PAX5 reactivity. This case was CD30-positive and CD15-positive, but also expressed CD3. Panels $\mathbf{c}$ (H\&E) and $\mathbf{d}$ (PAX5) feature a case of NLPHL that showed moderate PAX5 expression and the following immunophenotype: CD30-negative, CD20-weak positive, CD15-negative and epithelial membrane antigen-negative (original magnification $\times 200)$.

clinically suspicious for metastatic carcinoma. Because a definitive diagnosis could not be rendered on the original biopsy despite a battery of immunohistologic studies including CD20, an open biopsy was subsequently performed, which led to the diagnosis of diffuse large B-cell lymphoma. Although the material in the needle core biopsy was scant, the addition of PAX5 may have suggested B-cell differentiation and obviated the need for a second procedure. The second case was from a maxillary mass in a 63-year-old Guatemalan man, which was originally diagnosed as an undifferentiated malignant neoplasm, consistent with highgrade sarcoma. Unfortunately, no follow-up information is available for that patient. These two cases suggest that there is clinical utility in adding PAX5 to an antibody panel used for undifferentiated malignant neoplasms. A definitive diagnosis at the time of needle core biopsy, or, indeed at all, can be clinically advantageous in directing appropriate patient therapy.

We also document four cases of B-cell lymphomas that showed equivocal staining for CD20. The subsequent detection of PAX5 expression in these lymphomas allowed their assignment to lymphomas of the B lineage: PAX5 expression led to revision of the diagnosis as 'diffuse large B-cell lymphoma' in three cases (cecal mass in a 49-year-old male, base of tongue mass in a 76-year-old male and bone marrow aspirate in a 68-year-old female) and 'mediastinal large B-cell lymphoma' in the fourth case (mediastinal mass in a 34-year-old male). Three of these patients had no history of lymphoma and had not received anti-CD20 (Rituximab) chemotherapy. The fourth patient had been diagnosed with diffuse large B-cell lymphoma and myelofibrosis in a bone marrow biopsy 7 months previously and had received chemotherapy that included Rituximab. Because this latter patient had not previously presented with lymphadenopathy and had carried a diagnosis of myelofibrosis, the possibility of other diagnoses (such as hairy cell leukemia, since the patient had a history of splenomegaly requiring splenectomy) was entertained. In this latter case, the bone marrow biopsy showed an infiltrate of round lymphocytes admixed with a spindled cell proliferation, raising the possibility of a hematopoietic tumor associated with a mast cell disorder. The stain for CD20 highlighted only the round lymphoid cells, but the PAX5 stain highlighted both the round and 
spindled cell populations, indicating a morphologically biphasic B-cell lymphoma.

Our results show that robust staining for PAX5 is present in some B-cell lymphomas that lack or show equivocal staining for other pan B-cell markers and is in keeping with previous studies. ${ }^{9}$ The crisp nuclear staining of PAX5 is often better localized and more readily detected, particularly in small biopsies or fine needle aspirates in comparison to the staining for CD20 or CD79a. Since PAX5 expression is found in B-cell lymphomas arising from a broad differentiation spectrum and its loss in B-cell malignancies (unlike CD20) appears to be minimal, we advocate using PAX5 to establish B-cell identity. In addition, its expression at the protein level is not affected by anti-CD20 antibody (Rituximab) therapy, which renders it superior to CD20 for post-therapy detection of minimal residual disease.

We have additionally found PAX5 to be a useful marker in the diagnosis of Hodgkin lymphoma with atypical immunophenotypic features. Typically, CHL shows weaker PAX5 expression in Hodgkin cells than in background B lymphocytes. ${ }^{7,25,26}$ Several researchers have described a drastic loss of the B-cell-specific gene expression program in Hodgkin/Reed-Sternberg cells that may be related to downregulation of PAX5 mRNA. ${ }^{27,28}$ This downregulation of gene expression most likely corresponds at the phenotypic level to decreased PAX5 protein expression and to less intense staining in tissue biopsies. The downregulation may also correspond to increased expression of non-B-lineage markers often detected in CHL that may confound its diagnosis. ${ }^{29,30}$ These studies further underscore the important role of PAX5 in B-lineage identity and support our findings that staining for PAX5 serves as a useful marker in the diagnosis of CHL. Of note, we have also reported PAX5 expression in a series of cutaneous Hodgkin lymphoma, a finding that can prove useful in the distinction between cutaneous CHL and other CD30-positive lymphoproliferative disorders common to the skin. ${ }^{31}$

Despite reports of PAX5 mRNA expression in medulloblastoma, neuroblastoma and astrocytomas, ${ }^{12,14,32}$ we find no evidence of PAX5 protein expression by immunohistochemistry in these and many other epithelial and mesenchymal tumors. Less than $1 \%$ of over 300 bladder tumors in this study showed reactivity with the PAX5 antibody, a frequency significantly less than that reported for PAX5 mRNA expression in bladder tumors. ${ }^{10,11}$ Although it is possible that PAX5 protein expression in these tumors is below the level of detection by immunohistochemistry, it is also likely that additional post-transcriptional mechanisms are at play in the regulation of PAX5 protein expression that may account for this discrepancy.

The observed frequency of PAX5 staining in Merkel cell carcinoma in this series $(3 / 17$ cases, or $18 \%$ ) is less than that previously reported by Dong et $a l^{33}$ (29 of 31 cases) and Torlakovic et al ${ }^{34}$ (two of two cases). PAX5 staining was not detected in seven additional neuroendocrine tumors from various sites. These differences may be the consequence of variable fixation and processing techniques or antigen retrieval; however, we caution that PAX5 should be used as part of an immunohistologic panel that includes other hematopoietic and nonhematopoietic markers for tumors with small, round blue cell morphology to avoid tumor misclassification.

In summary, we have reported increased diagnostic yield in the study of undifferentiated neoplasms by adding PAX5 to a panel of lineage-specific antibodies. PAX5 is also of diagnostic utility in establishing B-lineage identity in both classical and NLPHLs that show equivocal CD20 staining or have unusual immunophenotypes. Finally, we have presented a large array of epithelial and mesenchymal tumors that lack PAX5 staining and, with the important exceptions of rare Merkel cell and bladder carcinomas, we have demonstrated the relatively high specificity of PAX5 expression for B cells.

\section{Acknowledgement}

This work was supported in part by Grant funding NIH CA34322.

\section{Disclosure/conflict of interest}

The authors declare no disclosures or conflicts of interest.

\section{References}

1 Palmisano WA, Crume KP, Grimes MJ, et al. Aberrant promoter methylation of the transcription factor genes PAX5 alpha and beta in human cancers. Cancer Res 2003;63:4620-4625.

2 Schafer BW. Emerging roles for PAX transcription factors in cancer biology. Gen Physiol Biophys 1998;17: 211-224.

3 Adams B, Dorfler P, Aguzzi A, et al. Pax-5 encodes the transcription factor BSAP and is expressed in B lymphocytes, the developing CNS, and adult testis. Genes Dev 1992;6:1589-1607.

4 Hagman J, Wheat W, Fitzsimmons D, et al. Pax-5/ BSAP: regulator of specific gene expression and differentiation in B lymphocytes. Curr Top Microbiol Immunol 2000;245:169-194.

5 Barr FG. Chromosomal translocations involving paired box transcription factors in human cancer. Int J Biochem Cell Biol 1997;29:1449-1461.

6 Lin P, Mahdavy M, Zhan F, et al. Expression of PAX5 in CD20-positive multiple myeloma assessed by immunohistochemistry and oligonucleotide microarray. Mod Pathol 2004;17:1217-1222.

7 Torlakovic E, Torlakovic G, Nguyen PL, et al. The value of anti-Pax-5 immunostaining in routinely fixed and paraffin-embedded sections: a novel pan pre-B and B-cell marker. Am J Surg Pathol 2002;26:1343-1350. 
8 Zhang X, Lin Z, Kim I. Pax5 expression in nonHodgkin's lymphomas and acute leukemias. J Korean Med Sci 2003;18:804-808.

9 Dong HY, Liu Z, Browne P, et al. B-cell malignancies lacking common pan B-cell antigens consistently express PAX-5. Mod Pathol 2004;17(Suppl 1):246A.

10 Adshead JM, Ogden CW, Penny MA, et al. The expression of PAX5 in human transitional cell carcinoma of the bladder: relationship with de-differentiation. BJU Int 1999;83:1039-1044.

11 Babjuk M, Soukup V, Mares J, et al. The expression of PAX5, p53 immunohistochemistry and p53 mutation analysis in superficial bladder carcinoma tissue. Correlation with pathological findings and clinical outcome. Int Urol Nephrol 2002;34:495-501.

12 Baumann Kubetzko FB, Di Paolo C, Maag C, et al. The $P A X 5$ oncogene is expressed in N-type neuroblastoma cells and increases tumorigenicity of a S-type cell line. Carcinogenesis 2004;25:1839-1846.

13 Gerard M, Abitbol M, Delezoide AL, et al. PAX-genes expression during human embryonic development, a preliminary report. C R Acad Sci III 1995;318:57-66.

14 Kozmik Z, Sure U, Ruedi D, et al. Deregulated expression of PAX5 in medulloblastoma. Proc Natl Acad Sci USA 1995;92:5709-5713.

15 Urbanek P, Fetka I, Meisler MH, et al. Cooperation of Pax 2 and Pax 5 in midbrain and cerebellum development. Proc Natl Acad Sci USA 1997;94:5703-5708.

16 Jaffe ES, Harris NL, Stein H, et al. (eds). World Health Organization classification of tumours. Pathology and genetics of tumours of haematopoietic and lymphoid tissues. IARC Press: Lyon, 2001, pp 351.

17 Kononen J, Bubendorf L, Kallioniemi A, et al. Tissue microarrays for high-throughput molecular profiling of tumor specimens. Nat Med 1998;4:844-847.

18 Natkunam Y, Mason DY. Prognostic immunohistologic markers in human tumors: why are so few used in clinical practice? Lab Invest 2006;86:742-747.

19 Alizadeh AA, Eisen MB, Davis RE, et al. Distinct types of diffuse large B-cell lymphoma identified by gene expression profiling. Nature 2000;403:503-511.

20 Lossos IS, Morgensztern D. Prognostic biomarkers in diffuse large B-cell lymphoma. J Clin Oncol 2006;24: 995-1007.

21 Shaffer AL, Wright G, Yang L, et al. A library of gene expression signatures to illuminate normal and pathological lymphoid biology. Immunol Rev 2006;210:67-85.

22 Morrison AM, Nutt SL, Thevenin C, et al. Loss- and gain-of-function mutations reveal an important role of BSAP (Pax-5) at the start and end of B cell differentiation. Semin Immunol 1998;10:133-142.

23 Rolink AG, Schaniel C, Andersson J, et al. Selection events operating at various stages in B cell development. Curr Opin Immunol 2001;13:202-207.

24 Nutt SL, Heavey B, Rolink AG, et al. Commitment to the B-lymphoid lineage depends on the transcription factor Pax5. Nature 1999;401:556-562.

25 Browne P, Petrosyan K, Hernandez A, et al. The B-cell transcription factors BSAP, Oct-2, and BOB.1 and the pan-B-cell markers CD20, CD22, and CD79a are useful in the differential diagnosis of classic Hodgkin lymphoma. Am J Clin Pathol 2003;120:767-777.

26 Krenacs L, Himmelmann AW, Quintanilla-Martinez L, et al. Transcription factor B-cell-specific activator protein (BSAP) is differentially expressed in B cells and in subsets of B-cell lymphomas. Blood 1998;92: 1308-1316.

27 Hertel CB, Zhou XG, Hamilton-Dutoit SJ, et al. Loss of B cell identity correlates with loss of B cell-specific transcription factors in Hodgkin/Reed-Sternberg cells of classical Hodgkin lymphoma. Oncogene 2002;21: 4908-4920.

28 Schwering I, Brauninger A, Klein U, et al. Loss of the B-lineage-specific gene expression program in Hodgkin and Reed-Sternberg cells of Hodgkin lymphoma. Blood 2003;101:1505-1512.

29 Nutt SL, Morrison AM, Dorfler P, et al. Identification of BSAP (Pax-5) target genes in early B-cell development by loss- and gain-of-function experiments. EMBO J 1998;17:2319-2333.

30 Uehira K, Amakawa R, Ito T, et al. A Hodgkin's disease cell line, KM-H2, shows biphenotypic features of dendritic cells and B cells. Int J Hematol 2001;73: 236-244.

31 Balzer BL, Harvell JD, Natkunam Y. Cutaneous Hodgkin lymphoma: an analysis of six cases. Mod Pathol 2004;17:89A.

32 Stuart ET, Kioussi C, Aguzzi A, et al. PAX5 expression correlates with increasing malignancy in human astrocytomas. Clin Cancer Res 1995;1:207-214.

33 Dong HY, Liu W, Cohen P, et al. B-cell specific activation protein encoded by the PAX-5 gene is commonly expressed in Merkel cell carcinoma and small cell carcinomas. Am J Surg Pathol 2005;29: 687-692.

34 Torlakovic E, Slipicevic A, Robinson C, et al. Pax-5 expression in nonhematopoietic tissues. Am J Clin Pathol 2006;126:798-804. 